\title{
The onset of superconductivity in long rectangles
}

\author{
Y. ALMOG \\ Faculty of Mathematics, Technion - Israel Institute of Technology, Haifa 32000, Israel
}

(Received 9 January 2002; revised 5 February 2002)

\begin{abstract}
The linearized Ginzburg-Landau equations in both semi-infinite strips and rectangles are transformed into equivalent one-dimensional integral equations. Then, the properties of the integral equations are utilized to prove that the onset field for a semi-infinite strip is isolated. We solve the integral equations numerically to obtain the onset field for both rectangles and semi-infinite strips. A formal asymptotic expansion of the onset field in the long rectangle limit is also obtained. Using this formal expansion, we show that the onset field converges in this limit faster than any finite exponential rate, and as a byproduct, that the onset mode in a semi-infinite strip must be asymptotically symmetric.
\end{abstract}

\section{Introduction}

Consider a planar superconducting body which is placed in a sufficiently low temperature (below the critical one) under the action of an external magnetic field. It is known both from experiments [17] and rigorous analysis [12] that, for a sufficiently strong magnetic field, the normal state prevails. If the field is then decreased, there is a critical field, depending on the sample's geometry, where the material enters the superconducting state. For samples with boundaries, this field is known as the onset field. We shall refer to the bifurcating mode of the super-conducting order parameter as the onset mode.

The simplest case in which the bifurcation from the normal state to the superconducting one was calculated is the case of a half-plane [20]. Even in this simple case the onset field is substantially larger than the bifurcation field on the real line [11]. Furthermore, it was found by Saint-James \& De-Gennes [20] that superconductivity is concentrated in this case near the boundary. This phenomenon, which appears in the presence of boundaries, has therefore been termed surface superconductivity. The significance of Saint-James \& de Gennes' [20] solution extends far beyond the simple, one-dimensional example of a half-plane. It was proved, first for films [6] then for disks [3], and finally for general two-dimensional domains with smooth boundaries [16, 10], that as the domain's scale tends to infinity, the onset field tends to de-Gennes' value. If the boundaries include wedges the onset field will be larger than de-Gennes' value [5, 14, 21, 13].

The Ginzburg-Landau energy functional may be represented in the following dimensionless form [9]:

$$
E=\int\left(-|\psi|^{2}+\frac{|\psi|^{4}}{2}+|\boldsymbol{H}|^{2}+\left|\frac{1}{\kappa} \nabla \psi-i \boldsymbol{A} \psi\right|^{2}\right) d x d y
$$


in which $\Psi$ is the (complex) superconducting order parameter, such that $|\Psi|$ varies from $|\Psi|=0$ (when the material is in the normal state) to $|\Psi|=1$ (for the purely superconducting state). The magnetic vector potential is denoted by $\boldsymbol{A}$ (the magnetic field is then given by $\boldsymbol{H}=\boldsymbol{\nabla} \times \boldsymbol{A}$ ), and $\kappa$ is the Ginzburg-Landau parameter which is a material property. Superconductors for which $\kappa<1 / \sqrt{2}$ are termed type I superconductors, and those for which $\kappa>1 / \sqrt{2}$ are termed type II. Note that $E$ is invariant under the gauge transformation

$$
\Psi \rightarrow e^{i \kappa \theta} \psi ; \quad \boldsymbol{A} \rightarrow \boldsymbol{A}+\nabla \theta .
$$

We look for local minimizers of $E$ in the semi-infinite strip $\mathscr{S}$ or in the rectangle $R_{L}$, in the the case where the applied magnetic field is constant and perpendicular to the plane. The Euler-Lagrange equations associated with $E$ (the steady state Ginzburg-Landau equations) are given by

$$
\begin{aligned}
& \left(\frac{i}{\kappa} \nabla+\boldsymbol{A}\right)^{2} \Psi=\Psi\left(1-|\Psi|^{2}\right), \\
& -\nabla \times(\nabla \times A)=\frac{i}{2 \kappa}\left(\Psi^{*} \nabla \Psi-\Psi \nabla \Psi^{*}\right)+|\Psi|^{2} A .
\end{aligned}
$$

The natural boundary conditions satisfied on $\partial \mathscr{S}$ for this problem are

$$
\begin{aligned}
\left(\frac{i}{\kappa} \nabla+\boldsymbol{A}\right) \psi \cdot \hat{\boldsymbol{n}} & =0, \\
\boldsymbol{H} & =h \hat{z} .
\end{aligned}
$$

As the Ginzburg-Landau equations are gauge-invariant, we may choose the gauge (following $[1,8]) \boldsymbol{A}=(0, A(x, y), 0)$. Thus, $\boldsymbol{H}=(0,0, H(x, y))$ and $H=\partial A / \partial x$. We then linearize (1.3) near the normal state $\Psi \equiv 0, A=h x$, to which end we assume the asymptotic expansions, for $\epsilon \ll 1$,

$$
\begin{aligned}
\Psi & =\epsilon^{1 / 2} \psi, \\
A & =h x+\epsilon a, \\
h & =h^{(0)}+\epsilon h^{(1)}+\ldots, \\
a & =a^{(0)}+\epsilon a^{(1)}+\ldots, \\
\psi & =\psi^{(0)}+\epsilon \psi^{(1)}+\ldots
\end{aligned}
$$

Applying the transformations

$$
x^{1}=\kappa x, \quad y^{1}=\kappa y, \quad h^{1}=\frac{h^{(0)}}{\kappa},
$$

the linearized form of (1.3a) becomes (we omit the superscripts 1 and (0) in the following)

$$
-\left(\frac{\partial^{2} \psi}{\partial x^{2}}+\frac{\partial^{2} \psi}{\partial y^{2}}\right)+2 i h x \frac{\partial \psi}{\partial y}=\psi-h^{2} x^{2} \psi
$$


and the boundary conditions become

$$
(i \nabla+h x \boldsymbol{j}) \cdot \boldsymbol{n}=0 .
$$

In the present contribution, we focus on semi-infinite strips and long rectangles. In earlier work [2], some relevant results were obtained for these domains. Let $\mathscr{S}$ denote the semi-infinite strip $\{(x, y) \mid-l \leqslant x \leqslant l, 0 \leqslant y\}$ and let $R_{L}$ denote the rectangle $\{(x, y) \mid-l \leqslant x \leqslant l, 0 \leqslant y \leqslant L\}$. Denote further by $h_{\mathscr{S}}$ the onset field of $\mathscr{S}$, by $h_{L}$ the onset field of $R_{L}$, and by $h_{1 D}$ the onset field of the interval $[-l, l]$. By the onset field we mean the largest value of $h$ for which non-trivial solutions exist to (1.6 $a)$, together with (1.6b).

The following relevant results were proved in Almog [2]:

(1) $h_{\mathscr{S}}$ exists and is greater than $h_{1 D}$.

(2) The onset mode of $\mathscr{S}$ decays exponentially fast as $y \rightarrow \infty$, or is $O\left(e^{-\gamma y}\right)$. The value of $\gamma$ can be easily obtained once $h_{\mathscr{S}}$ is determined.

(3) $h_{L} \geqslant h_{\mathscr{S}}$. Furthermore, $h_{L} \underset{L \rightarrow \infty}{\longrightarrow} h_{\mathscr{S}}$.

Many of the results in Almog [2] were obtained using Fourier analysis. In the present contribution, we employ Fourier analysis once again to obtain integral equations equivalent to the bifurcation problems in $\mathscr{S}$ and $R_{L}$. We then utilize the properties of the equation in $\mathscr{S}$ to prove isolation, on the $h$ axis, of $h_{\mathscr{S}}$. We then provide formal arguments, showing that $h_{L}$ converges faster than any exponential rate to $h_{\mathscr{S}}$ as $L \rightarrow \infty$, and that the onset mode in $\mathscr{S}$ is asymptotically symmetric with respect to $x$ as $y \rightarrow \infty$. Finally, we obtain $h_{L}$ and $h_{\mathscr{S}}$ by numerically solving the formerly derived integral equations. Once $h_{\mathscr{S}}$ is obtained, the exponential rate of decay of the onset mode in $\mathscr{S}$, for large $y$, is evaluated.

The rest of this contribution is arranged as follows. In the next section we derive integral equations equivalent to $(1.6 a)$ together with $(1.6 b)$ in both $\mathscr{S}$ and $R_{L}$. In $\S 3$ we prove isolation of $h_{\mathscr{S}}$, in $\S 4$ we show, using formal arguments, that $h_{L} \sim h_{\mathscr{S}}+O\left(e^{-\lambda L}\right)$ for any $\lambda>0$, and that $\psi(x, y) \sim \bar{\psi}(-x, y)$ as $y \rightarrow \infty$. Finally, in $\S 5$, we solve the integral equation from $\S 2$ numerically.

\section{Integral equations}

Theorem 1 Let $h=h_{\mathscr{S}}$ and let $\mathscr{H}=\mathscr{C}^{\epsilon}(\mathscr{S}) \bigcap \mathscr{W}^{\epsilon, \infty}(\mathscr{S})$. Then, any solution of the problem

$$
\begin{gathered}
-\left(\frac{\partial^{2} \psi}{\partial x^{2}}+\frac{\partial^{2} \psi}{\partial y^{2}}\right)+2 i h x \frac{\partial \psi}{\partial y}=\psi-h^{2} x^{2} \psi \quad-l \leqslant x \leqslant l, \quad 0 \leqslant y, \\
\psi_{x}( \pm l, y)=0, \quad i \psi_{y}(x, 0)+h x \psi(x, 0)=0
\end{gathered}
$$

in $\mathscr{H}$ must satisfy

$$
\frac{1}{2} \psi(x, 0)=\frac{1}{2 \pi} P V \int_{-\infty}^{\infty} i \int_{-l}^{l}(h s-\omega) G(x, s, \omega) \psi(s, 0) d s d \omega,
$$


where P.V denotes the principal value and

$G(x, s, \omega)=m(h)\left\{\begin{array}{c}\frac{\left[U(\eta) U^{\prime}(-\xi(l))+U(-\eta) U^{\prime}(\xi(l))\right]\left[U^{\prime}(-\xi(-l)) U(\xi)+U^{\prime}(\xi(-l)) U(-\xi)\right]}{U^{\prime}(\xi(-l)) U^{\prime}(-\xi(l))-U^{\prime}(\xi(l)) U^{\prime}(-\xi(-l))}, \\ \frac{\left[U(\eta) U^{\prime}(-\xi(-l))+U(-\eta) U^{\prime}(\xi(-l))\right]\left[U^{\prime}(-\xi(l)) U(\xi)+U^{\prime}(\xi(l)) U(-\xi)\right]}{U^{\prime}(\xi(-l)) U^{\prime}(-\xi(l))-U^{\prime}(\xi(l)) U^{\prime}(-\xi(-l))}, \\ x>s,\end{array}\right.$

wherein

$$
m(h)=\frac{\Gamma(1 / 2+a)}{2 \sqrt{\pi h}}
$$

$\xi=\xi(x, \omega)$ is given by

$$
\xi=\sqrt{\frac{2}{h}}(h x-\omega),
$$

and $\eta=\xi(s, \omega), U(x) \equiv U(a, x)$ is a parabolic cylinder function of the first kind, and $a=-\frac{1}{2 h}$.

Conversely, suppose that for some $h=\tilde{h}_{\mathscr{S}}, \exists f \in C^{2}[-l, l]$ satisfying $f^{\prime}( \pm l)=0$ and

$$
\frac{1}{2} f(x)=\frac{1}{2 \pi} P V \int_{-\infty}^{\infty} \hat{\phi}(x, \omega) d \omega
$$

where

$$
\hat{\phi}(x, \omega)=i \int_{-l}^{l}(h s-\omega) G(x, s, \omega) f(s) d s .
$$

Then, $\tilde{h}_{\mathscr{S}} \leqslant h_{\mathscr{S}}$. Furthermore, if $\tilde{h}_{\mathscr{S}}=h_{\mathscr{S}}$, then the function

$$
\phi(x, y)=\frac{1}{2 \pi} P V \int_{-\infty}^{\infty} e^{i \omega y} \hat{\phi}(x, \omega) d \omega
$$

is a solution of (2.1)

Proof Suppose first that $\psi \in \mathscr{H}$ satisfies (2.1). (Recall that in Almog [2] it is shown that there exist a solution of (2.1) which decays exponentially fast as $y \rightarrow \infty$, and hence the set of solutions in $\mathscr{H}$ is by no means an empty set.) Upon multiplying (2.1a) by $\exp \{-i \omega y\}$ and integrating by parts we obtain that

$$
\hat{\psi}(x, \omega)=\int_{0}^{\infty} e^{-i \omega y} \psi(x, y) d y
$$

satisfies

$$
\begin{aligned}
& -\hat{\psi}^{\prime \prime}+\left[(h x-\omega)^{2}-1\right] \hat{\psi}=i(h x-\omega) \psi(x, 0) \\
& \hat{\psi}^{\prime}( \pm l)=0
\end{aligned}
$$


where $\hat{\psi}^{\prime}=\partial \hat{\psi} / \partial x$. Hence,

$$
\hat{\psi}(x, \omega)=i \int_{-l}^{l}(h s-\omega) G(x, s, \omega) \psi(s, 0) d s .
$$

Note that $G(x, s, \omega)$ is well defined since the denominator in $(2.3 a)$ does not vanish for all $\omega \in \mathbb{R}$ when $h>h_{1 D}$ (cf. $[8,5,2]$ ). Integrating $(2.6)$ by parts we obtain

$$
\hat{\psi}(x, \omega) \sim \frac{\psi(x, 0)}{\omega}+O\left(\frac{1}{\omega^{2}}\right) .
$$

Hence, the inverse Fourier transform of $\hat{\psi}$ exists and is given by

$$
\frac{1}{2 \pi} \mathrm{PV} \int_{-\infty}^{\infty} e^{i \omega y} \hat{\psi}(x, \omega) d \omega= \begin{cases}\psi(x, y), & y>0, \\ \frac{1}{2} \psi(x, 0), & y=0, \\ 0, & y<0\end{cases}
$$

Hence,

$$
\frac{1}{2} \psi(x, 0)=\frac{1}{2 \pi} \mathrm{PV} \int_{-\infty}^{\infty} \hat{\psi}(x, \omega) d \omega .
$$

Substituting (2.8) together with (2.10) into (2.1b) we obtain (2.2).

Suppose now that for some $h \geqslant h_{\mathscr{S}}, \exists f \in C^{2}[-l, l]$ satisfying $f^{\prime}( \pm l)=0$ and (2.4). In Almog [2] it was demonstrated that

$$
\begin{aligned}
G(x, s, \omega)= & G(x, s, \omega, h)=\frac{1}{|\omega|}\left[G_{1}(x, s, \omega, h) e^{-|\omega||x-s|}\right. \\
& \left.+G_{2}(x, s, \omega, h) e^{-|\omega|(2 l-x-s)}+G_{3}(x, s, \omega, h) e^{-|\omega|(2 l+x+s)}\right],
\end{aligned}
$$

where, as $\omega \rightarrow \infty$,

$$
\begin{aligned}
& G_{1}=|\omega| \frac{1}{2} \frac{(\omega-h s)^{a-1 / 2}}{(\omega-h x)^{a+1 / 2}} \exp \left\{\frac{1}{2} h\left(s^{2}-x^{2}\right)\right\}\left[1+O\left(\frac{1}{\omega^{2}}\right)\right], \\
& G_{2}=|\omega| \frac{1}{2} \frac{(\omega-h l)^{2 a}}{(\omega-h x)^{a+1 / 2}(\omega-h s)^{a+1 / 2}} \exp \left\{\frac{1}{2} h\left(2 l^{2}-s^{2}-x^{2}\right)\right\}\left[1+O\left(\frac{1}{\omega^{2}}\right)\right], \\
& G_{3}=|\omega| \frac{1}{2} \frac{(\omega+h l)^{2 a}}{(\omega-h x)^{a+1 / 2}(\omega-h s)^{a+1 / 2}} \exp \left\{\frac{1}{2} h\left(2 l^{2}-s^{2}-x^{2}\right)\right\}\left[1+O\left(\frac{1}{\omega^{2}}\right)\right],
\end{aligned}
$$

whenever $x<s$. For the cases $x>s$ and $\omega \rightarrow-\infty$ we use the identities

$$
G(x, s, \omega, h)=G(s, x, \omega, h)=G(-x,-s,-\omega, h) .
$$

Hence, applying Laplace's method (cf. [4, 18], for instance) it is not difficult to show that for $|\omega| \gg 1$

$$
\hat{\phi}(x, \omega) \sim-i \frac{f(x)}{\omega}-i h x \frac{f(x)}{\omega^{2}}+O\left(\frac{1}{\omega^{3}}\right) .
$$

As $\hat{\phi}$ satisfies

$$
\begin{aligned}
& -\hat{\phi}^{\prime \prime}+\left[(h x-\omega)^{2}-1\right] \hat{\phi}=i(h x-\omega) f(x) \\
& \hat{\phi}^{\prime}( \pm l)=0
\end{aligned}
$$


and since by $(2.13) f^{\prime}( \pm l)=0$, the function

$$
\varphi=\hat{\phi}+i \frac{f(x)}{\omega}
$$

must satisfy

$$
\begin{aligned}
& -\varphi^{\prime \prime}+\left[(h x-\omega)^{2}-1\right] \varphi=-i h x f+\frac{i}{\omega}\left[\left(h^{2} x^{2}-1\right) f-f^{\prime \prime}\right], \\
& \varphi^{\prime}( \pm l)=0 .
\end{aligned}
$$

Hence,

$$
\varphi(x, \omega)=\int_{-l}^{l} G(x, s, \omega)\left\{-i h s f+\frac{i}{\omega}\left[\left(h^{2} s^{2}-1\right) f-f^{\prime \prime}\right]\right\} d s,
$$

from which we can deduce the $O\left(\omega^{-3}\right)$ term in the asymptotic expansion of $\hat{\phi}(x, \omega)$ :

$$
\hat{\phi}(x, \omega) \sim-i \frac{f(x)}{\omega}-i h x \frac{f(x)}{\omega^{2}}-i \frac{\left(h^{2} x^{2}+1\right) f+f^{\prime \prime}}{\omega^{3}}+O\left(\frac{1}{\omega^{4}}\right) .
$$

Substituting the above expansion into (2.14a) yields

$$
\hat{\phi}^{\prime \prime}(x, \omega) \sim-i \frac{f^{\prime \prime}(x)}{\omega}+O\left(\frac{1}{\omega^{2}}\right),
$$

and hence

$$
\hat{\phi}^{\prime}(x, \omega) \sim-i \frac{f^{\prime}(x)}{\omega}+O\left(\frac{1}{\omega^{2}}\right) .
$$

Consider then the function $\phi(x, y)$ defined in (2.5). By $(2.13) \phi(x, y)$ is differentiable twice in $x$ and $y$ for any $y \neq 0$. Furthermore,

$$
\begin{gathered}
\phi\left(x, 0^{+}\right)-\phi\left(x, 0^{-}\right)=f(x), \\
\frac{\partial \phi}{\partial y}\left(x, 0^{+}\right)-\frac{\partial \phi}{\partial y}\left(x, 0^{-}\right)=i h x f(x) .
\end{gathered}
$$

By (2.17), (2.18), (2.19), and (2.20) the various derivatives of $\phi$ are given by

$$
\begin{gathered}
\phi_{x}(x, y)=\frac{1}{2 \pi} \mathrm{PV} \int_{-\infty}^{\infty} e^{i \omega y} \hat{\phi}^{\prime}(x, \omega) d \omega, \\
\phi_{x x}(x, y)=\frac{1}{2 \pi} \mathrm{PV} \int_{-\infty}^{\infty} e^{i \omega y} \hat{\phi}^{\prime \prime}(x, \omega) d \omega, \\
\phi_{y}(x, y)=\frac{1}{2 \pi} \mathrm{PV} \int_{-\infty}^{\infty} e^{i \omega y}[i \omega \hat{\phi}(x, \omega)-f(x)] d \omega, \\
\phi_{y y}(x, y)=\frac{1}{2 \pi} \mathrm{PV} \int_{-\infty}^{\infty} e^{i \omega y}\left[-\omega^{2} \hat{\phi}(x, \omega)-i(h x+\omega) f(x)\right] d \omega .
\end{gathered}
$$

Obviously, by (2.14b) we have

$$
\phi_{x}( \pm l)=0 \quad \forall y \neq 0 .
$$

Furthermore, by (2.21) we have

$$
-\left(\frac{\partial^{2} \phi}{\partial x^{2}}+\frac{\partial^{2} \phi}{\partial y^{2}}\right)+2 i h x \frac{\partial \phi}{\partial y}=\phi-h^{2} x^{2} \phi, \quad-l \leqslant x \leqslant l, \quad y \neq 0 .
$$


It remains yet to check the conditions satisfied by $\phi$ for $y=0^{+}$. As, by (2.4a),

$$
\frac{1}{2}\left[\phi\left(x, 0^{+}\right)+\phi\left(x, 0^{-}\right)\right]=\frac{1}{2 \pi} \mathrm{PV} \int_{-\infty}^{\infty} \hat{\phi}(x, \omega) d \omega=\frac{1}{2} f(x),
$$

combination with (2.20a) yields

$$
\phi\left(x, 0^{-}\right)=0
$$

Hence, for $y<0, \phi(x, y)$ is a solution of (2.23) and (2.22) together with the Dirichlet boundary condition $\phi(x, 0)=0$.

Suppose now that the problem in the lower semi-infinite strip $x \in[-l, l], y<0$ admits only the trivial solution. Then, as $\phi_{y}\left(x, 0^{-}\right)=0$, we have by (2.20)

$$
\phi_{y}\left(x, 0^{+}\right)=i h x \phi\left(x, 0^{+}\right)
$$

and the theorem follows. Hence, it remains necessary to show that no solution other than the trivial one exists to (2.23) and (2.22) for $y<0$ together with $\phi(x, 0)=0$.

Applying the transformation $y \rightarrow-y \phi \rightarrow \phi^{*}$, the problem for $y<0$ becomes identical with the same problem for $y>0$. Then let

$$
\begin{gathered}
\lambda(h)=\inf _{\substack{\psi \in H^{1}(\mathscr{S}, \mathbb{C}) \\
\|\psi\|_{L^{2}(\mathscr{S})}=1}} \int_{\mathscr{S}}|(\nabla-i h x \hat{\boldsymbol{j}}) \psi|^{2} d x d y, \\
\lambda_{D}(h)=\inf _{\substack{\psi \in H^{1}(\mathscr{G}, \mathbb{C}) \\
\|\psi\|_{L^{2}(\mathscr{S})}=1 ; \psi(x, 0)=0}} \int_{\mathscr{S}}|(\nabla-i h x \hat{\boldsymbol{j}}) \psi|^{2} d x d y .
\end{gathered}
$$

Suppose first that $h>h_{\mathscr{S}}$. Then, $\lambda_{D}(h) \geqslant \lambda(h)>1$ (cf. Almog [2]), and hence no nontrivial solutions exist to (2.22), (2.23), and $\phi(x, 0)=0$ (cf. Bauman et al. [3]).

Hence, the upper critical field of (2.4) $\tilde{h}_{\mathscr{S}}$ must be exactly $h_{\mathscr{S}}$. Furthermore, let $h=h_{\mathscr{S}}$. In this case $\lambda\left(h_{\mathscr{S}}\right)=1$ and hence $\lambda_{D}\left(h_{\mathscr{S}}\right) \geqslant 1$. If $\lambda_{D}\left(h_{\mathscr{S}}\right)>1$ then $\phi(x, y) \equiv 0$ for $y<0$, and hence (2.5) is indeed a solution of (2.1). Even if $\lambda_{D}\left(h_{\mathscr{S}}\right)=1$ no nontrivial solution can exist to (2.22), (2.23), and $\phi(x, 0)=0$, otherwise it must be the minimizer of the quadratic forms on the right-hand side of both (2.27a) and (2.27b), and as no solution but the trivial one can satisfy both (2.25) and (2.26) on $y=0$, the theorem is proved.

In addition to the integral equation, it is possible to derive similar integral equations for the rectangle $R_{L}=\{(x, y) \mid-l \leqslant x \leqslant l ; 0 \leqslant y \leqslant L\}$ as the following theorem states:

Theorem 2 Let $h=h_{L}$. Then, any solution of the problem

$$
\begin{aligned}
& -\left(\frac{\partial^{2} \psi^{L}}{\partial x^{2}}+\frac{\partial^{2} \psi^{L}}{\partial y^{2}}\right)+2 i h x \frac{\partial \psi^{L}}{\partial y}=\psi^{L}-h^{2} x^{2} \psi^{L}, \quad-l \leqslant x \leqslant l, 0 \leqslant y, \\
& \psi_{x}^{L}( \pm l, y)=0, \quad i \psi_{y}^{L}(x, 0)+h_{L} x \psi^{L}(x, 0)=i \psi_{y}^{L}(x, L)+h_{L} x \psi^{L}(x, L)=0
\end{aligned}
$$


must satisfy

$$
\begin{gathered}
-\frac{1}{2}\left[\psi^{L}(x, 0)+\psi^{L}(x, L)\right]=\frac{1}{L} P V \sum_{n=-\infty}^{\infty} \hat{\psi}_{L}(x, n \chi), \\
-\frac{1}{2}\left[\psi^{L}(x, L)-\psi^{L}(x, 0)\right]=\frac{1}{L} P V \sum_{n=-\infty}^{\infty} \tilde{\psi}_{L}(x,(n+1 / 2) \chi),
\end{gathered}
$$

where $\chi=2 \pi / L$ and

$$
\begin{gathered}
\hat{\psi}_{L}(x, \omega)=-i \int_{-l}^{l}(h s-\omega) G(x, s, \omega)[\psi(s, L)-\psi(s, 0)] d s, \\
\tilde{\psi}_{L}(x, \omega)=i \int_{-l}^{l}(h s-\omega) G(x, s, \omega)[\psi(s, 0)+\psi(s, L)] d s .
\end{gathered}
$$

Conversely, suppose for some $h \exists f, g \in C^{2}[-l, l]$ satisfying $f^{\prime}( \pm l)=g^{\prime}( \pm l)=0$ and

$$
\begin{gathered}
-\frac{1}{2} g=\frac{1}{L} P V \sum_{n=-\infty}^{\infty} \hat{\phi}_{L}(x, n \chi), \\
-\frac{1}{2} f=\frac{1}{L} P V \sum_{n=-\infty}^{\infty} \tilde{\phi}_{L}(x,(n+1 / 2) \chi),
\end{gathered}
$$

where

$$
\begin{gathered}
\hat{\phi}_{L}(x, \omega)=-i \int_{-l}^{l}(h s-\omega) G(x, s, \omega) f(s) d s, \\
\tilde{\phi}_{L}(x, \omega)=i \int_{-l}^{l}(h s-\omega) G(x, s, \omega) g(s) d s .
\end{gathered}
$$

Then, $h \leqslant h_{L}$. Furthermore, if $h=h_{L}$, then the function

$$
\phi^{L}(x, y)=\frac{1}{L} P V \sum_{n=-\infty}^{\infty} \hat{\phi}^{L}(x, n \chi) e^{i n \gamma y}
$$

is a solution of (2.28)

Proof The proof of (2.30) is very similar to the proof of (2.2) in Theorem 1. Interested readers can find the details in Almog [2]. The converse statement is proved as follows. Let

$$
\begin{gathered}
\varphi^{L}(x, y)=\frac{1}{L} \mathrm{PV} \sum_{n=-\infty}^{\infty} \tilde{\phi}^{L}(x, n \chi) e^{i n x y}, \\
\Psi^{L}=\phi^{L}+\varphi^{L} e^{\frac{1}{2} i x y} .
\end{gathered}
$$

Following the same steps used in the proof of the converse statement in Theorem 1 it is not difficult to show that

$$
\begin{gathered}
-\left(\frac{\partial^{2} \Psi^{L}}{\partial x^{2}}+\frac{\partial^{2} \Psi^{L}}{\partial y^{2}}\right)+2 i h x \frac{\partial \Psi^{L}}{\partial y}=\Psi^{L}-h^{2} x^{2} \Psi^{L}, \quad-l \leqslant x \leqslant l, 0 \leqslant y \\
\psi_{x}^{L}( \pm l, y)=0, \quad \psi^{L}(x, 0)=\Psi^{L}(x, L)=0 .
\end{gathered}
$$


One can then define

$$
\begin{gathered}
\lambda^{L}(h)=\inf _{\substack{\psi \in H^{1}\left(R_{L}, \mathbb{C}\right) \\
\|\psi\|_{L^{2}\left(R_{L}\right)}=1}} \int_{R_{L}}|(\nabla-i h x \hat{\boldsymbol{j}}) \psi|^{2} d x d y, \\
\lambda_{D}^{L}(h)=\inf _{\substack{\psi \in H^{1}\left(R_{L}, \mathbb{C}\right) \\
\|\psi\|_{L^{2}\left(R_{L}\right)}=1 ; \psi(x, 0)=\psi(x, L)=0}} \int_{R_{L}}|(\nabla-i h x \hat{\boldsymbol{j}}) \psi|^{2} d x d y .
\end{gathered}
$$

As $R_{L}$ is compact, $\lambda^{L}<\lambda_{D}^{L}$, and consequently, non-trivial solutions for (2.35) can exist only if $h<h_{L}$. Let then $h \geqslant h_{L}$ and suppose that the system (2.31) is satisfied by appropriate $f$ and $g$. Then, by (2.32a) and (2.33) we have

$$
\begin{gathered}
\phi^{L}(x, L)-\phi^{L}(x, 0)=f(x), \\
\frac{\partial \phi^{L}}{\partial y}(x, L)-\frac{\partial \phi^{L}}{\partial y}(x, 0)=i h x f(x),
\end{gathered}
$$

and hence

$$
\left.\phi_{y}^{L}(x, y)\right|_{y=0} ^{L}=\left.i h x \phi^{L}(x, y)\right|_{y=0} ^{L} .
$$

As $\Psi_{L} \equiv 0$ for $h \geqslant h_{L}$ we have

$$
\phi_{y}(x, 0)+\phi_{y}(x, L)=\left.\left[\varphi_{y}^{L}(x, y)+\frac{1}{2} i \chi \varphi(x, y)\right]\right|_{y=0} ^{L} .
$$

However, by (2.32b) and (2.34),

$$
\begin{gathered}
\varphi^{L}(x, L)-\varphi^{L}(x, 0)=g(x) \\
\frac{\partial \varphi^{L}}{\partial y}(x, L)-\frac{\partial \varphi^{L}}{\partial y}(x, 0)=i\left(h x-\frac{1}{2} \chi\right) g(x) .
\end{gathered}
$$

Since by $(2.31 \mathrm{a})$

$$
\phi(x, 0)+\phi(x, L)=g .
$$

\section{Isolation of the onset field}

In the following we demonstrate an application of Theorem 1: we show that the onset field $h_{\mathscr{S}}$ for the semi-infinite strip $\mathscr{S}$ is isolated. Explicitly we prove the following result:

Theorem 3 There exists $\delta>0$ such that $\forall h \in\left(h_{\mathscr{S}}-\delta, h_{\mathscr{S}}\right)$ (2.1) is satisfied by the trivial solution only.

It should first be noted that in some cases, the onset field is not isolated. Consider, for instance, the onset problem for an infinite strip

$$
\begin{gathered}
-\left(\frac{\partial^{2} \psi}{\partial x^{2}}+\frac{\partial^{2} \psi}{\partial y^{2}}\right)+2 i h x \frac{\partial \psi}{\partial y}=\psi-h^{2} x^{2} \psi, \quad-l \leqslant x \leqslant l, \quad y \in \mathbb{R}, \\
\psi_{x}( \pm l, y)=0 .
\end{gathered}
$$


Substituting the ansatz

$$
\psi(x, y)=F(x) e^{-i \omega y},
$$

we obtain

$$
F^{\prime \prime}-\left[(h x-\omega)^{2}-1\right] F=0, \quad F^{\prime}( \pm l)=0 .
$$

Let $h(\omega)$ denote the largest value of $h$, for given $\omega \in \mathbb{R}$, for which (3.3) admits non-trivial solutions. It can be shown (cf. Bolley \& Helffer [6]) that the onset field in this case,

$$
h_{1 D}=h\left(\omega_{0}\right)=\sup _{\omega \in \mathbb{R}} h(\omega) .
$$

In addition to (3.4) it was shown (cf. [6]) that $h(\omega)$ is continuous, and that for every $\omega \neq \pm \omega_{0} h(\omega) \neq h_{1 D}$. Hence, $h(\omega)$ is not constant near $\omega_{0}$ and therefore, $h_{1 D}$ is not isolated.

To prove Theorem 3 we need the following auxiliary result:

Lemma 1 The integral operator $\mathscr{L}: \mathscr{C}[-\uparrow, \uparrow] \rightarrow \mathscr{C}[-\uparrow, \uparrow]$ defined by

$$
\mathscr{L}\left\{=\frac{\infty}{\in \pi} P V \int_{-\infty}^{\infty}\left\lceil\omega \int _ { - \uparrow } ^ { \uparrow } \left(\langle \int - \omega ) \mathscr { G } ( \S , \int , \omega ) \left\{( \int ) \left\lceil\int\right.\right.\right.\right.\right.
$$

is compact, and holomorphic in $h$ for $h>h_{1 D}$.

Proof To prove the lemma we show that

$$
\mathscr{L}\left\{=\int_{-\uparrow}^{\uparrow} \mathscr{K}\left(\S, \int,\langle)\left\{( \int ) \left\lceil\int,\right.\right.\right.\right.
$$

where $K(x, s, h)$ is continuous in both $x$ and $s$ and holomorphic in $h$. To this end we write $\mathscr{L}$ in the form

$$
\mathscr{L}\left\{=\frac{\infty}{\in \pi} \int_{1}^{\infty}\left\lceil\omega \int _ { - \uparrow } ^ { \downarrow } \left[\left(\left\langle\int-\omega\right) \mathscr{G}\left(\S, \int, \omega\right)+\left(\left\langle\int+\omega\right) \mathscr{G}\left(\S, \int,-\omega\right)\right]\left\{( \int ) \left\lceil\int .\right.\right.\right.\right.\right.\right.
$$

We then apply to (3.7) the asymptotic expansion (2.11), together with the identity (2.12), to obtain, for $x \leqslant s$ and $\omega \gg 1$,

$$
\begin{aligned}
& (h s-\omega) G(x, s, \omega)+(h s+\omega) G(x, s,-\omega) \sim \\
& \quad \sinh \left\{\frac{h}{2}\left(x^{2}-s^{2}\right)\right\} e^{-\omega(s-x)}+\frac{1}{\omega}\left\{(s-x) F_{1}(x, s) e^{-\omega(s-x)}+\right. \\
& \left.+(2 l-s-x) F_{2}(x, s) e^{-\omega(2 l-s-x)}+(2 l+s+x) F_{3}(x, s) e^{-\omega(2 l+s+x)}\right\}+O\left(\omega^{-2}\right) .
\end{aligned}
$$

The exact form of the functions $F_{1}, F_{2}$ and $F_{3}$ can be obtained, but need not concern us since they are all bounded. Similar expansion can be obtained for $x \geqslant s$.

By (3.8),

$$
\int_{0}^{\infty}\left[(h s-\omega) G(x, s, \omega)+(h s+\omega) G(x, s,-\omega)-\sinh \left\{\frac{h}{2}\left(x^{2}-s^{2}\right)\right\} e^{-\omega(s-x)}\right] d \omega
$$


converges uniformly. The monotone convergence theorem guarantees, therefore, that

$$
\begin{aligned}
\int_{0}^{\infty} d \omega \int_{-l}^{x}[(h s-\omega) G(x, s, \omega)+(h s+\omega) G(x, s,-\omega)] f(s) d s \\
=\int_{-l}^{x} d s \int_{0}^{\infty}[(h s-\omega) G(x, s, \omega)+(h s+\omega) G(x, s,-\omega)] f(s) d \omega .
\end{aligned}
$$

A similar equality can be derived for $x \geqslant s$.

Then let

$$
K(x, s, h)=\int_{0}^{\infty}[(h s-\omega) G(x, s, \omega)+(h s+\omega) G(x, s,-\omega)] d \omega .
$$

It is possible to express $K(x, s, h)$ in the form

$$
K(x, s, h)=K_{1}(x, s, h)+K_{2}(x, s, h),
$$

where

$$
K_{1}(x, s, h)= \begin{cases}\frac{\sinh \left\{\frac{h}{2}\left(x^{2}-s^{2}\right)\right\}}{s-x} & x \neq s, \\ 0 & x=s,\end{cases}
$$

and $K_{2}(x, s, h)$ is continuous in view of the above-established uniform convergence.

To prove that $\mathscr{L}$ is holomorphic in $h$ we show that $K(x, s, h)$ is holomorphic in some neighbourhood of the real line for $h>h_{1 D}$. The size of this neighbourhood should be fixed for all $(x, s) \in[-l, l]^{2}$. Then, Theorem VII-2.6 in Kato [15] would prove that $\mathscr{L}$ is holomorphic as well.

Clearly, $K_{1}(x, s, h)$ is an entire function in $h$ for all $x$ and $s$. Inasmuch as ( $h s-$ $\omega) G(x, s, \omega)+(h s+\omega) G(x, s,-\omega)$ is holomorphic for $h>h_{1 D}$, and in view of the uniform convergence of the integral (3.9), $K_{2}(x, s, h)$ is holomorphic as well for $h>h_{1 D}$.

To complete the proof of Theorem 3 we consider the equation

$$
\mathscr{L}(\langle)\{=\lambda\{\text {. }
$$

By Theorem 1, non-trivial solutions to (3.13) would exist for $h=h_{\mathscr{S}}, \lambda=1 / 2$. By Lemma 1 this eigenvalue has finite multiplicity, and hence, since $\mathscr{L}$ is holomorphic and by Theorem VII-1.9 in Kato [15] we obtain that $\lambda=1 / 2$ is satisfied either

(1) for all $h>h_{1 D}$, or

(2) for a finite set of values in $\left[h_{1 D}+\delta, h_{\mathscr{S}}\right]$ for any $\delta>0$.

However, (2.4) does not admit any non-trivial solutions for $h>h_{\mathscr{S}}$ by Theorem 1. Hence, the second option is the viable one, which proves Theorem 3.

\section{The onset field in the long rectangle limit}

It was demonstrated in [2] that the onset field of the rectangle $R_{L}$ tends to the onset field of the semi-infinite strip $\mathscr{S}$, or that $h_{L} \rightarrow h_{\mathscr{S}}$. In the following we attempt to derive the next order term of the asymptotic behaviour in that limit. To this end, we first derive a 
new set of integral equations in replace of (2.4) and (2.28), which, in contrast to the latter set of equations, is real and self-adjoint.

Consider first the problem in $\mathscr{S}$. It is not difficult to show, using integration by parts, that

$$
\frac{1}{2 \pi} \mathrm{PV} \int_{-\infty}^{\infty} e^{i \omega y}[i \omega \hat{\psi}(x, \omega)-\psi(x, 0)] d \omega= \begin{cases}\frac{\partial \psi(x, y)}{\partial y}, & y>0, \\ \frac{\partial}{2} \frac{\partial \psi(x, y)}{\partial y}(x, 0), & y=0, \\ 0, & y<0 .\end{cases}
$$

Substituting the above identity, (2.8), and (2.9) into (2.1c) we obtain

$$
\frac{1}{2 \pi} \mathrm{PV} \int_{-\infty}^{\infty}\left[(h x-\omega) \int_{-l}^{l} G(x, s, \omega)(h s-\omega) \psi(s, 0) d s-\psi(x, 0)\right] d \omega=0 .
$$

In a similar manner, we can obtain the equation

$$
\frac{1}{L} \mathrm{PV} \sum_{n=-\infty}^{\infty}\left[\left.(h x-n \chi) \int_{-l}^{l} G(x, s, n \chi)(h s-n \chi) \psi(s, y)\right|_{0} ^{L} d s-\left.\psi(x, y)\right|_{0} ^{L}\right]=0
$$

for the problem in $R_{L}$.

Equation (4.2), though complicated, has a major advantage on (2.4): it is a real selfadjoint equation, and is, therefore, the most natural way to present (2.1) in Fourier space. Obviously, (4.2) must have real solutions, for if $f(x)$ is a solution then so is $\bar{f}(x)$. The fact that if $f(x)$ is a solution of (2.4) then $\bar{f}(x)$ is not, may seem puzzling but can be easily resolved. It is not difficult to show, following the same procedure used to prove Theorem 1 , that if $f(x)$ is a solution of (4.2), then

$$
\phi(x, y)=\frac{1}{2 \pi} \mathrm{PV} \int_{-\infty}^{\infty} e^{i \omega y} \int_{-l}^{l} i(h s-\omega) G(x, s, \omega) f(s) d s d \omega
$$

is a solution of (2.1). However, if we substitute $f(x)=\bar{\psi}(x, 0)$, where $\psi(x, y)$ is a solution of (2.1), we obtain $\phi(x, y)=-\bar{\psi}(x,-y)$, or equivalently

$$
\phi(x, y)= \begin{cases}0, & y>0, \\ \frac{1}{2} \bar{\psi}(x, 0), & y=0, \\ -\bar{\psi}(x,-y), & y<0 .\end{cases}
$$

We notice that $\phi\left(x, 0^{-}\right) \neq 0$, which explains why $\phi\left(x, 0^{-}\right)$cannot solve (2.4). Consequently, there are solutions of (4.2) which do not satisfy (2.4), that when substituted into (4.5) yield solution $\phi(x, y)$ of (2.1) for which $\phi\left(x, 0^{+}\right) \neq f(x)$. Hence, keeping the above in mind, we can treat (4.2) as a real self-adjoint equation which is equivalent to (2.1).

We seek an approximation for a solution $\left(f_{L}(x), h_{L}\right)$ of (4.3). We expect that the leading order term, as $L \rightarrow \infty$, would be a solution of (4.2). Hence, it is necessary to approximate the difference between the left-hand-sides of (4.2) and (4.3) in that limit.

In order to approximate the above difference we first rewrite (4.3) in the form

$$
\frac{1}{L} \sum_{n=-\infty}^{\infty}\left[\hat{\phi}_{L}(x, n \chi)+\hat{\phi}_{L}(x,-n \chi)\right]=0,
$$


wherein

$$
\hat{\phi}_{L}(x, \omega)=\left(h_{L} x-\omega\right) \int_{-l}^{l}\left(h_{L} s-\omega\right) G(x, s, \omega) f_{L}(s) d s-f_{L}(x) .
$$

It is not difficult to show, using (2.11), that

$$
\Phi_{L}(x, \omega)+\Phi_{L}(x,-\omega) \sim-\frac{h^{2} x^{2}}{\omega^{2}} f_{L}(x)
$$

and that

$$
\frac{\partial \Phi_{L}}{\partial \omega}(x, \omega)-\frac{\partial \Phi_{L}}{\partial \omega}(x,-\omega) \sim \frac{2 h^{2} x^{2}}{\omega^{3}} f_{L}(x)
$$

as $\omega \rightarrow \infty$. Hence,

$$
\int_{-\infty}^{\infty}\left|\Phi_{L}(x, \omega)+\Phi_{L}(x,-\omega)\right|+\left|\frac{\partial \Phi_{L}}{\partial \omega}(x, \omega)-\frac{\partial \Phi_{L}}{\partial \omega}(x,-\omega)\right| d \omega<\infty .
$$

Consequently, $\Phi_{L}(x, \omega)+\Phi_{L}(x,-\omega)$ is both absolutely integrable and of bounded variation in $\omega$, and thus, one can apply the Poisson summation formula to obtain

$$
\frac{1}{L} \mathrm{PV} \sum_{n=-\infty}^{\infty} \hat{\phi}_{L}(x, n \chi)=\frac{1}{2 \pi} \sum_{n=-\infty}^{\infty} \mathrm{PV} \int_{-\infty}^{\infty} e^{-i \omega n L} \hat{\phi}_{L}(x, \omega) d \omega .
$$

The function $\hat{\phi}_{L}(x, \omega)$ is holomorphic in $\omega$ except for a countable number of points where

$$
U^{\prime}(\xi(-l, \omega)) U^{\prime}(-\xi(l, \omega))-U^{\prime}(\xi(l, \omega)) U^{\prime}(-\xi(-l, \omega))=0 .
$$

For $h>h_{1 D}$ there exist a strip of analyticity of $\hat{\phi}_{L}(x, \omega)$, $\Im \omega<\gamma$, where the above equation is not satisfied [2]. Hence,

$$
\mathrm{PV} \int_{-\infty}^{\infty} e^{-i \omega n L} \hat{\phi}_{L}(x, \omega) d \omega \sim O\left(e^{-\gamma n L}\right) \quad \text { as } L \longrightarrow \infty .
$$

Consequently, we can obtain the following approximate form of (4.3):

$$
\mathrm{PV} \int_{-\infty}^{\infty} \hat{\phi}_{L}(x, \omega) d \omega \cong-\mathrm{PV} \int_{-\infty}^{\infty} e^{-i \omega L}\left[\hat{\phi}_{L}(x, \omega)+\hat{\phi}_{L}(x,-\omega)\right] d \omega+O\left(e^{-2 \gamma L}\right) .
$$

As the right-hand side of (4.13) is of $O\left(e^{-\gamma L}\right)$ we substitute into both sides the formal asymptotic expansion

$$
\begin{aligned}
& h_{L}=h_{\mathscr{S}}+e^{-\gamma L} h^{1}(L)+O\left(e^{-2 \gamma L}\right), \\
& f_{L}=f_{\mathscr{S}}+e^{-\gamma L} f^{1}(L)+O\left(e^{-2 \gamma L}\right),
\end{aligned}
$$

where $h^{1}(L)$ and $f^{1}(L)$ are $O(1)$ as $L \rightarrow \infty$. Let

$$
\hat{\phi}_{\mathscr{S}}(x, \omega)=i\left(h_{\mathscr{S}} x-\omega\right) \int_{-l}^{l}\left(h_{\mathscr{S} S}-\omega\right) G(x, s, \omega) f_{\mathscr{S}}(s) d s-f_{\mathscr{S}}(x) .
$$

Using the same complex plane techniques applied in [2], it is not difficult to show that

$$
\int_{-\infty}^{\infty} \hat{\phi}_{\mathscr{S}}(x, \omega) e^{i \omega L} d \omega \cong 2 \pi i e^{-\gamma L}\left[e^{i \alpha L} \operatorname{Res}\left\{\omega_{+}, \hat{\phi}_{\mathscr{S}}\right\}+e^{-i \alpha L} \operatorname{Res}\left\{\omega_{-}, \hat{\phi}_{\mathscr{S}}\right\}\right]+O\left(e^{-2 \gamma L}\right),
$$


where $\omega_{+}=\alpha+i \gamma$ and $\omega_{-}=-\alpha+i \gamma$ are the closest points to the real axis in the upper half of the complex plane where (4.11) is satisfied (in the case $\alpha=0$ which is expected to take place for sufficiently small $l$ we have $\omega_{+}=\omega_{-}$). The following assumptions were made while deriving (4.16):

(1) There are at most two poles of $\hat{\phi}_{\mathscr{S}}(x, \omega)$ on the line $\mathfrak{\Im} \omega=\gamma$.

(2) Either $\operatorname{Res}\left\{\omega_{+}, \hat{\phi}_{\mathscr{S}}\right\} \neq 0$ or $\operatorname{Res}\left\{\omega_{-}, \hat{\phi}_{\mathscr{S}}\right\} \neq 0$.

If assumption 1 is not satisfied it would be necessary to introduce additional terms into the right-hand-side of (4.16) corresponding to the additional roots of (4.11). If assumption 2 is violated, higher order terms must be obtained.

In addition to the above assumptions it is convenient to assume that both $\omega_{+}$and $\omega_{-}$ are simple poles of $\hat{\phi}_{\mathscr{S}}$, an assumption which can be proved in the limit $l \rightarrow \infty$. In [2] it is demonstrated that

$$
\operatorname{Res}\left\{\omega_{+}, \hat{\psi}\right\}=A C_{+} g(x),
$$

where

$$
\begin{gathered}
g(x)=U\left(\xi\left(x, \omega_{0}^{+}\right)\right) U^{\prime}\left(\xi\left(l,-\omega_{0}^{+}\right)\right)+U\left(-\xi\left(x, \omega_{0}^{+}\right)\right) U^{\prime}\left(-\xi\left(l,-\omega_{0}^{+}\right)\right), \\
C_{+}=\int_{-l}^{l} g(s)\left(h_{\mathscr{S} S}-\omega_{+}\right) f_{\mathscr{S}}(s) d s,
\end{gathered}
$$

and $A$ is a constant which can be determined in principle. The other residue is given by

$$
\operatorname{Res}\left\{\omega_{-}, \hat{\psi}\right\}=\bar{A} \bar{C}_{-} \bar{g}(-x)
$$

where

$$
C_{-}=\int_{-l}^{l} g(s)\left(h_{\mathscr{S}} s-\omega_{+}\right) f_{\mathscr{S}}(-s) d s .
$$

Substituting (4.14)-(4.17) into (4.13), and applying the transformation $\omega \rightarrow h \omega$ to the integration dummy variable, we obtain, by equating terms of $O\left(e^{-\gamma L}\right)$,

$$
\begin{aligned}
\mathrm{PV} \int_{-\infty}^{\infty} & {\left[(x-\omega) \int_{-l}^{l} h_{\mathscr{S}}^{3} G(x, s, \omega)(s-\omega) f^{1}(s) d s-h_{\mathscr{S}} f^{1}(x)\right] d \omega } \\
= & i\left\{A e^{i \alpha L}\left[\left(h_{\mathscr{S}} x-\omega_{+}\right) C_{+} g(x)+\left(h_{\mathscr{S}} x+\omega_{+}\right) C_{-} g(-x)\right]\right. \\
& \left.-\bar{A} e^{-i \alpha L}\left[\left(h_{\mathscr{S}} x-\omega_{-}\right) \bar{C}_{-} \bar{g}(-x)+\left(h_{\mathscr{S}} x+\omega_{-}\right) \bar{C}_{+} \bar{g}(x)\right]\right\} \\
& -h^{1} \mathrm{PV} \int_{-\infty}^{\infty}\left\{(x-\omega) \int_{-l}^{l} \frac{\partial}{\partial h}\left[h^{3} G(x, s, \omega)\right]_{h=h_{\mathscr{S}}}(s-\omega) f_{\mathscr{S}}(s) d s-f_{\mathscr{S}}(x)\right\} d \omega .
\end{aligned}
$$

Multiplying by $f_{\mathscr{S}}(-x)$ and integrating over $[-l, l]$ yields, after a few manipulations, the solvability condition

$$
\begin{array}{r}
h^{1} \mathrm{PV} \int_{-\infty}^{\infty} \int_{-l}^{l}\left\{(x-\omega) \int_{-l}^{l} \frac{\partial}{\partial h}\left[h^{3} G(x, s, \omega)\right]_{h=h_{\mathscr{S}}}(s-\omega) f_{\mathscr{S}}(s) d s-f_{\mathscr{S}}(x)\right\} \\
\times f_{\mathscr{S}}(-x) d x d \omega=0 .
\end{array}
$$

It seems plausible to assume here that $h^{1}=0$ as there is no particular reason to believe that 
the integral it multiplies vanishes. Furthermore, multiplying (4.8) by $f_{\mathscr{S}}(x)$ and integrating we obtain

$$
\begin{aligned}
& h^{1} \mathrm{PV} \int_{-\infty}^{\infty} \int_{-l}^{l}\left\{(x-\omega) \int_{-l}^{l} \frac{\partial}{\partial h}\left[h^{3} G(x, s, \omega)\right]_{h=h_{\mathscr{S}}}(s-\omega) f_{\mathscr{S}}(s) d s-f_{\mathscr{S}}(x)\right\} \\
& \times f_{\mathscr{S}}(x) d x d \omega=i\left[A e^{i \alpha L}\left(C_{+}^{2}-C_{-}^{2}\right)-\bar{A} e^{-i \alpha L}\left(\bar{C}_{+}^{2}-\bar{C}_{-}^{2}\right)\right],
\end{aligned}
$$

and hence, if the left-hand side of (4.20) does not vanish, there exists $L$ such that $h_{L}<h_{\mathscr{S}}$ which contradicts a result which was rigorously proved in [2]. The above identity, thus, leads us to another important conclusion: $C_{+}=C_{-}$. Recall that the asymptotic behaviour of $\psi$ in $\mathscr{S}$ as $y \rightarrow \infty$ is

$$
\psi \sim C e^{-\gamma y}\left[C_{+} g(x) e^{i \alpha y}+\overline{C_{-} g(-x) e^{i \alpha y}}\right],
$$

and hence $\psi(x, y) \sim \bar{\psi}(-x, y)$. In other words we can say that $\psi$ is asymptotically symmetric.

If indeed, as we expect, $h^{1}=0$, then repeating the above procedure for higher order terms yields

$$
h_{L}-h_{\mathscr{S}} \sim o\left(e^{-} \lambda L\right)
$$

for any $\lambda>0$. Thus, obtaining the leading asymptotic behaviour is a difficult task which is left to future research.

\section{Numerical solution}

We conclude by presenting a numerical solution of (2.31). To achieve this end, we first need the estimates

$$
\frac{1}{L} \sum_{n=N+1}^{\infty}[\hat{\phi}(x, n \chi)+\hat{\phi}(x,-n \chi)] \cong-\frac{i h x}{\pi \chi} S_{1}(N) f+O\left(\frac{1}{N^{3} \chi^{3}}\right) \text { as } N \rightarrow \infty
$$

and

$$
\frac{1}{L} \sum_{n=N+1}^{\infty}[\hat{\phi}(x,(n+1 / 2) \chi)+\hat{\phi}(x,-(n+1 / 2) \chi)] \cong-\frac{i h x}{\pi \chi} S_{2}(N) g+O\left(\frac{1}{N^{3} \chi^{3}}\right) \text { as } N \rightarrow \infty,
$$

where

$$
\begin{gathered}
S_{1}(N)=\sum_{n=N+1} \frac{1}{n^{2}}=\frac{\pi^{2}}{6}-\sum_{n=1}^{N} \frac{1}{n^{2}}, \\
S_{2}(N)=\sum_{n=N+1} \frac{1}{(n+1 / 2)^{2}}=\frac{\pi^{2}}{2}-4-\sum_{n=1}^{N} \frac{1}{(n+1 / 2)^{2}} .
\end{gathered}
$$


For $N \chi$ sufficiently large we may, thus, rewrite (2.31) in the approximate form

$$
\begin{gathered}
\frac{1}{2} g=\frac{i}{L} \sum_{n=-N}^{N} \int_{-l}^{l}(h s-n \chi) G(x, s, n \chi) f(s) d s-\frac{i h x}{\pi \chi} S_{1}(N) f, \\
\frac{1}{2} f=\frac{i}{L} \sum_{n=-N-1}^{N} \int_{-l}^{l}(h s-(n+1 / 2) \chi) G(x, s,(n+1 / 2) \chi) g(s) d s-\frac{i h x}{\pi \chi} S_{2}(N) g .
\end{gathered}
$$

The solution of the above system was obtained by applying a quadrature method. For each $n$ the integrals in (5.2) were evaluated utilizing the trapezium rule except for a close neighbourhood of $x=s$. While it is still reasonable to assume $f(s) \approx f(x)$ for $s \in[x-\Delta, x+\Delta]$ it would be inaccurate to claim that $G(x, s, n \chi) \approx G(x, x, n \chi)$ in the same interval unless $n \chi \ll 1 / \Delta$ in view of (2.11). It is still possible, however, to use the approximation

$$
\int_{x-4}^{x+\Delta}(h s-n \chi) G(x, s, n \chi) f(s) d s \approx f(x) \int_{x-4}^{x+\Delta}(h s-n \chi) G(x, s, n \chi) d s,
$$

and to evaluate the integral on the right-hand-side using the trapezium rule with a finer grid (which should depend on $n$ ). We note that while the above approximation is $O(\Delta)$ accurate, it is not difficult to obtain $O\left(\Delta^{2}\right)$ accuracy by appropriately modifying it.

Once the matrices representing the integrals in (5.2) are calculated, we can represent the system in the form

$$
\begin{array}{r}
\sum_{k=-J}^{J} \sum_{j=-J}^{J}\left\{\left[K_{m j}^{(1)}(h)-\frac{i h x}{\pi \chi_{m}} S_{1}(N) \delta_{m j}\right]\left[K_{j k}^{(2)}(h)-\frac{i h x}{\pi \chi_{j}} S_{2}(N) \delta_{j k}\right]-\delta_{m k}\right\} g_{k}=0, \\
\forall-J \leqslant m \leqslant J,
\end{array}
$$

wherein $g_{k}=g(-l+k \Delta), \Delta=l / J$ and $K^{(1)}$ and $K^{(2)}$ respectively represent the integrals on (5.2a) and (5.2b).

Non-trivial solutions of (5.4) exist if and only if the determinant of the matrix multiplying the vector $\left[g_{-J}, \ldots, g_{J}\right]^{T}$ vanishes. We, therefore, look for the values of $h$ where this determinant vanishes, to which end we apply the Van Wijngaarden-Dekker-Brent Method [19]. The largest root is the onset field $h_{L}$. For sufficiently large $L$ this root is also an extremal point of the determinant and then Brent's method of minimization [19] was applied.

After the onset field has been found, (4.11) can be solved. The closest root to the real axis of this equation provides us with the asymptotic behaviour of $\psi$ in $\mathscr{S}$ as $y \rightarrow \infty$ according to (4.21). A globally convergent algorithm, based on the quasi-Newton method of minimization was applied [19].

Figure 1 displays the dependence of the various parameters on $l$ for the semi-infinite strip case. The solid line, the dashed line, and the dotted line respectively denote $h_{\mathscr{S}}(l)$, $\alpha(l)$, and $\gamma(l)(\alpha$ and $\gamma$ are defined immediately after (4.16)). The solution for this case was obtained by letting $L \rightarrow \infty$. Practically, $L=20$ was substituted for $l \leqslant 0.5, L=20 l$ was substituted for $0.5<l \leqslant 1$, and $L=10 l$ for $l>1$. In view of the fast exponential decay of $h_{L}-h_{\mathscr{S}}$ the error is expected to be vanishingly small as the numerical error is expected to be around $2-3 \%$. 


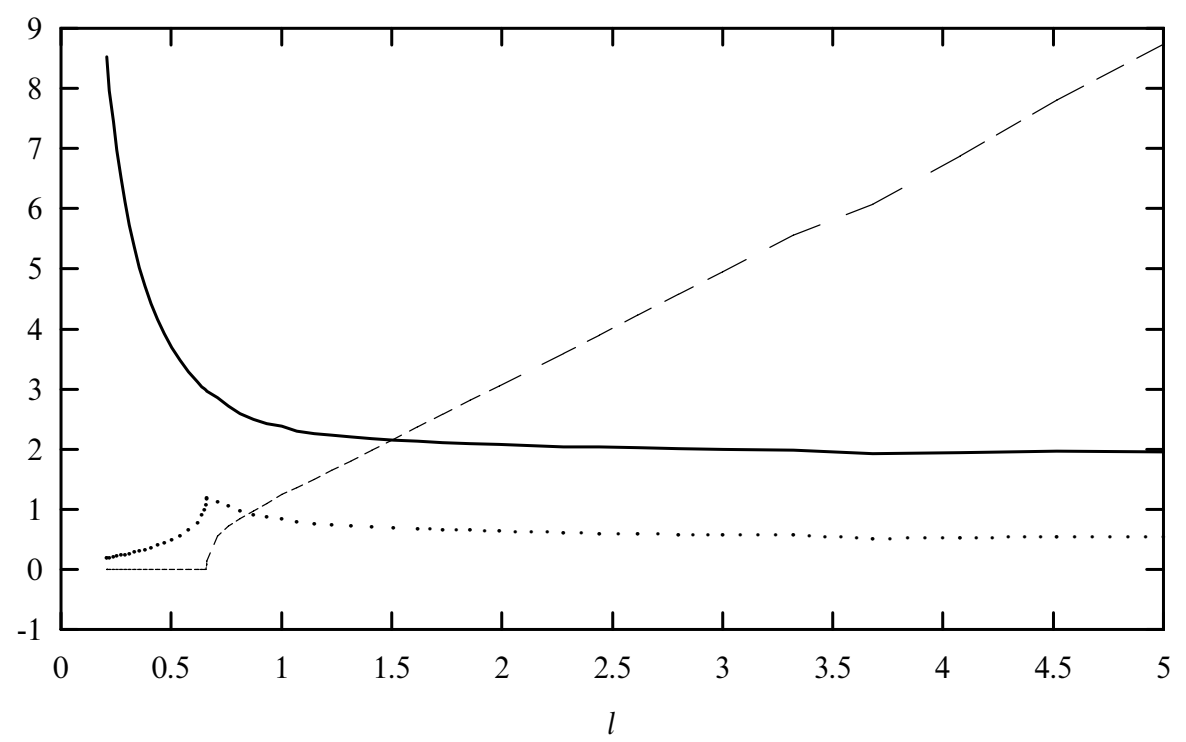

FIGURE 1. Dependence of $h_{\mathscr{S}}$ (denoted by the solid curve), $\gamma$ (denoted by the dotted curve), and $\alpha$ (denoted by the dashed curve) on $l$ for the semi-infinite strip case.

Figure 1 provides us with a few interesting observations:

(1) The asymptotic value of $h_{\mathscr{S}}$ in the limit $l \rightarrow \infty$, which is expected to be the onset field for the quarter plane $\{(x, y) \mid 0<x, y\}$, is approximately 1.96, in accordance with the calculations of Schweigert \& Peters [21], and in small disagreement with those of Jadallah et al. [14].

(2) There is a critical value of $l$ (denote it by $l_{c}$ ) such that for $l \leqslant l_{c} \alpha$ vanishes, but for $l>l_{c} \alpha$ is positive. Similar behaviour is observed in the infinite-strip (which is essentially one-dimensional) case, where for sufficiently small $l$ asymmetric modes cease to exist [6].

(3) The rate of decay of $\psi$ in $y$, or $\gamma$, reaches a maximum exactly at $l=l_{c}$. Apparently, the roots of (4.11) at $\omega_{+}$and $\omega_{-}$, which are simple for $l>l_{c}$ combine into a double root at $l=l_{c}$. Then, one of the roots travels downward along the imaginary axis.

(4) As $l \rightarrow \infty \alpha$ tends to be a linear function of $l$. It is not difficult to show that $U^{\prime}(-\xi(l, \omega))$ becomes exponentially small in this limit. Hence, $-\xi(l, \omega)$ must correspond to the root, which is the closest to the real line in the upper half plane, of $U^{\prime}$.

(5) As $l \rightarrow \infty h_{\mathscr{S}}$ tends to infinity, in accordance with results for thin films.

Figure 2 shows the dependence of $h_{L}$ on $L$ for two different values of $l$ : the thick curve corresponds to $l=0.5$ whereas the thin curve correspons to $l=1$. Note that as $L \rightarrow 0 h_{L}$ tends to infinity, which is again in accordance with results for the thin infinite strip and semi-infinite strip limits. In addition, one can observe that for $l=1 h_{L}$ is not monotonic, but attains a local maximum at $L \sim 3.2$ and a local minimum at $L \sim 2.8$. The minimum point, where the slope of the graph undergoes a jump discontinuity, takes place when a 


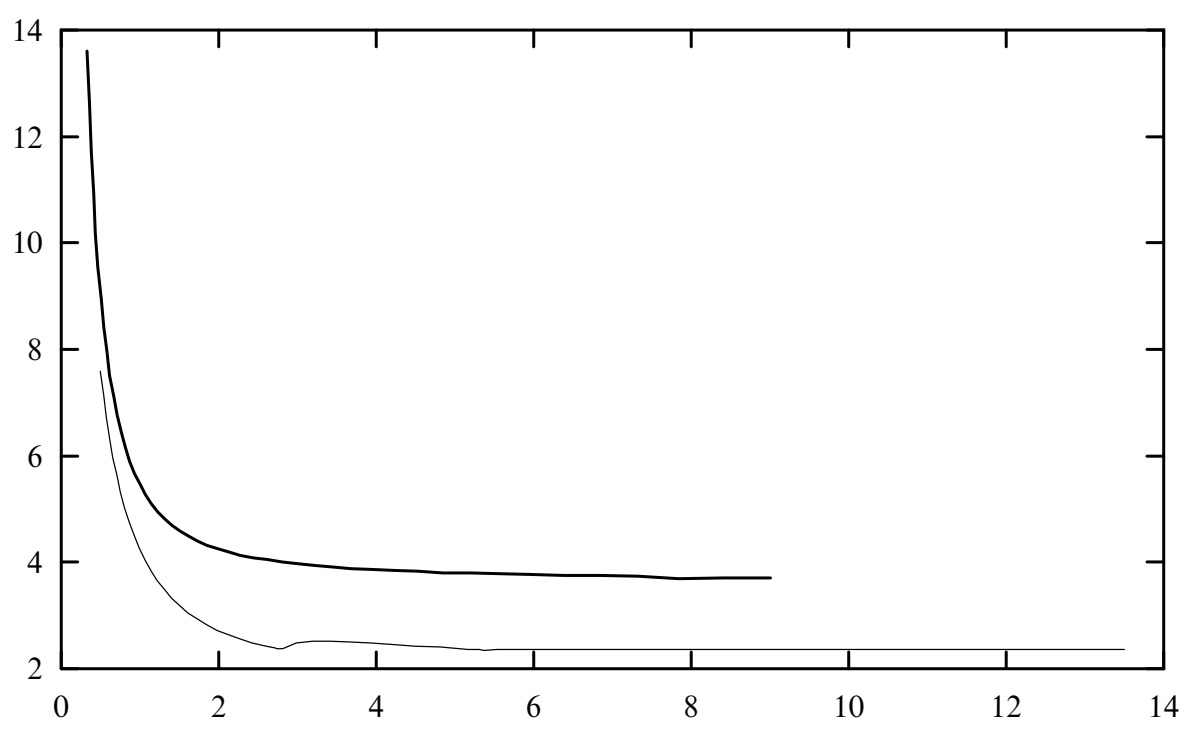

FIGURE 2. Dependence of $h_{L}$ on $l$ for the cases $l=1$, denoted by the thin curve, and the case $l=0.5$ denoted by the thick curve.

single vortex appears in the rectangle's center. The next discontinuity appears at $L \sim 5.35$, but can hardly be distinguished in the graph in view of the rapid decay of $h_{L}$ as $L \rightarrow \infty$.

To conclude this section, in Figure 3 we show two plots of the solution of (2.28) $\psi^{L}$ along the symmetry axis $x=0$. We note that, in all our numerical experiments, only symmetric solutions, i.e. $\psi^{L}(-x, y)=\psi^{L}(x, L-y)=\bar{\psi}(x, y)$, were found. The first plot, denoted by the thick curve, represents the case $l=0.64, L=12.8$, for which $\gamma \sim 0.868$, whereas the second plot, denoted by the thin curve, represents the case $l=1, L=20$, for which $\gamma \sim 0.812$. In both cases, $\left|\psi^{L}(0, y)\right|^{2} e^{2 \gamma y}$, normalized by its maximal value $\left(\max _{y \in[0, L / 2]}\left|\psi^{L}(0, y)\right|^{2} e^{2 \gamma y}\right.$ ), is plotted against $y / L$ for $0 \leqslant y \leqslant L / 2$. (In view of the symmetry there is no need to plot it for $L / 2<y \leqslant L$.)

The most obvious difference between the two modes of behaviour exhibited in Figure 3, is the oscillations of of the thin curve corresponding to the case $l=1$. The vortices are located in this case at $y / L=0.1185,0.2465,0.375$, and 0.5 . In this case $\pi /(\alpha L) \sim 0.126$, which is in good accordance with the location of the vortices, taking into account the contribution of the walls at $y=0, L$ and the numerical error. We suspect that the form of the last peak, at $y / L \sim 0.454$ is also affected by the numerical error which is magnified by a large factor of $e^{\gamma y} \sim 1500$ in this location.

Completely different is the case $l=0.64$ (which was preferred on the case $l=0.5$ in order to obtain a larger magnetic flux for the same value of $l / L$ ). In this case, a single vortex is located at $y=L / 2$. As $l<l_{c}$ in this case, we have $\alpha=0$, and hence no vortices are expected, except at $y=L / 2$, where the contribution of the walls at $y=0$ and $y=L$ cancel each other. Despite the relatively large magnetic flux through the rectangular sample (about $53 \%$ of the flux in the case $l=1$ ) the numerical data suggest that the vortex is simple. This result may be supported by the following argument: as

$$
\psi^{L}(0, y) \sim C\left[e^{-\gamma_{0} y}-e^{-\gamma_{0}(L-y)}\right]
$$




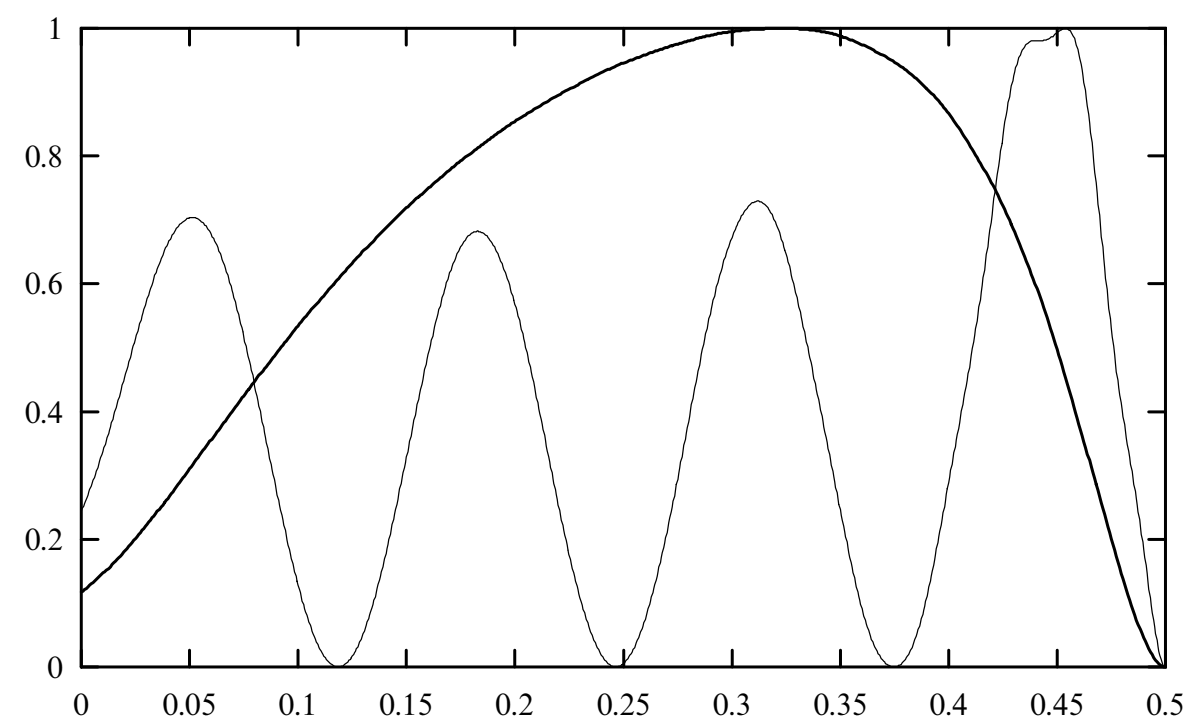

FIGURE 3. Plots of $\left|\psi_{L}(0, y)\right|^{2} e^{2 \gamma_{0} y}$ for $0 \leqslant y / L \leqslant 1 / 2$. The thick curve denotes the case $l=0.64, L=12.8$, whereas the thin curve denotes the case $l=1, L=20$.

for $y \gg 1$ and $L-y \gg 1$ in this case, $\nabla \psi \neq 0$ at $y=L / 2$, and hence, the vortex must be simple.

Finally, we note that for the infinite strip case it is well known that the no vortices can exist for sufficiently thin strips [7]. The same result is expected for semi-infinite strips since there is no symmetry point the vortex is 'pushed' in this case to infinity.

\section{Conclusion}

We have transformed, using Fourier analysis, in $\S 2$, the linearized Ginzburg-Landau equations in both semi-infinite strips and rectangles into an equivalent set of integral equations: we proved that the upper critical field of the integral equations coincides with the onset field for the relevant domain. These integral equations are by no means the most natural way to present the linearized Ginzburg-Landau equations in Fourier space, as they are non-self-adjoint. Nevertheless, we showed in $\S 3$ that the integral operators are of Fredholm type with kernels holomorphic in $h$. It was then possible to use these properties to show that the onset field in semi-infinite strips $h_{\mathscr{S}}$ is isolated, i.e. $\exists \delta>0: h \in\left(h_{\mathscr{S}}-\delta, h_{\mathscr{S}}\right) \Rightarrow$ no non-trivial solution exists.

The integral equations are also suitable for numerical solution. In $\S 5$ the onset field was calculated numerically by solving the integral equations for rectangles. The numerical scheme's main advantage is that it can be applied to arbitrarily long rectangles. When both the width and the length of the rectangle become large the method is still at least comparable with other techniques [21,14]. Once the onset field is found, it is possible to obtain the bifurcating solution and compare it with some of the theoretical predictions in [2].

In addition to the integral equations developed in $\S 2$, another set of integral equations 
were obtained in $\S 4$ for both rectangles and semi-infinite strips. These equations seem to be inapplicable for numerical purposes, nevertheless they are both real and self-adjoint, and hence, it was natural to apply them in the asymptotic expansion of the onset field in the long rectangle limit (where the length of one side is kept constant). It was found, by means of this formal expansion, that the onset field converges faster than any exponential rate to the onset field in the corresponding semi-infinite strip. Furthermore, it was demonstrated that the onset mode in a semi-infinite strip is asymptotically symmetric.

\section{Acknowledgements}

This research was supported by the Technion V.P.R fund - M. and C. Papo Research Fund, by the David Posnack Memorial Academic Lectureship and by the fund for the promotion of research at the Technion. The author wishes to thank Professor Yehuda Pinchover for many useful comments and discussions.

\section{References}

[1] Abrikosov, A. A. (1957) On the magnetic properties of superconductors of the second group. Soviet Phys. J.E.T.P. 5, 1175-1204.

[2] Almog, Y. (2000) The onset of superconductivity in semi-infinite strips. J. Math. Phys. 41, 7889-7905.

[3] Bauman, P., Philips, D. \& Tang, Q. (1998) Stable nucleation for the Ginzburg-Landau system with an applied magnetic field. Arch. Rat. Mech. Anal. 142, 1-43.

[4] Bennder, C. M. \& Orszag, S. M. (1978) Advanced Mathematical Methods for Scientists and Engineers - Asymptotic methods and perturbation theory, McGraw-Hill.

[5] Bernoff, A. \& Sternberg, P. (1998) Onset of superconductivity in decreasing fields for general domains. J. Math. Phys. 39, 1272-1284.

[6] Bolley, C. \& HelfFer, B. (1996) Rigorous results on Ginzburg-Landau models in a film submitted to exterior parallel magnetic field I. Nonlinear Studies, 3, 1-29.

[7] Bolley, C. \& Helffer, B. (1998) Stability of bifurcating solutions for the Ginzburg-Landau equations. Rev. Math. Phys. 10, 579-626.

[8] Chapman, S. J. (1994) Nucleation of superconductivity in decreasing fields I. Euro. J. Appl. Math. 5, 449-468.

[9] Chapman, S. J. (1995) Asymptotic analysis of the Ginzburg-Landau model of superconductivity: reduction to a free boundary model. Quart. J. Appl. Math. 53, 601-627.

[10] del Pino, M., Felmer, P. L. \& Sternberg, P. (2000) Boundary concentration for eigenvalue problems related to the onset of the superconductivity. Comm. Math. Phys., 210, 413-446.

[11] Ginzburg, V. L. \& Landau, L. D. (1950) On the theory of supercoductivity. Soviet Phys. JETP, 20, 1064.

[12] Giorgi, T. \& Philips, D. (1999) The breakdown of superconductivity due to strong fields for the Ginzburg-Landau model. SIAM J. Math. Anal. 30, 341-359.

[13] Jadallah, H. T. (n.d.) The onset of superconductivity in a domain with a corner. Preprint.

[14] Jadallah, H. T., Rubinstein, J. \& Sterenberg, P. (1999) Phase transition curves for mesoscopic superconucting samples. Phys. Rev. Lett. 82, 2935.-2938.

[15] Kato, T. (1980) Perturbation Theory for Linear Operators. 3rd ed. Springer-Verlag.

[16] LU, K. \& PAN, X. B. (1999) Eigenvalue problems of Ginzburg-Landau operator in bounded domains. J. Math. Phys. 50, 2647-2670.

[17] Meissner, W. \& OChSEnfeld, R. (1933) Naturwissenschaffen, 21, 787.

[18] Murray, J. D. (1984) Asymptotic Analysis. Springer-Verlag. 
[19] Numerical Recepies Software (1992) Numerical Recepies in Fortran 77. Cambridge University Press.

[20] Saint-James, D. \& DE Gennes, P. G. (1963) Onset of superconductivity in decreasing fields. Phys. Lett. 7, 306-308.

[21] Schweigert, V. A. \& Peeters, F. M. (1999) Influence of the confinement geometry on surface superconductivity. Phys. Rev. B, 60, 3084-3087. 